Año 11.

Revista de Investigación

Frontera

Académica sin

Núm. 28

ISSN: 2007-8870

http://revistainvestigacionacademicasinfrontera.com

Recibido el 2 de abril de 2018. Dictaminado mediante arbitraje favorablemente 30 de agosto de 2018

\title{
Tendencias actuales en ambientes de enseñanza-aprendizaje-conocimiento del uso de Tecnología de Información y Comunicación en entornos educativos presenciales y a distancia en las Instituciones de Educación Superior.
}

\section{Current trends in teaching-learning-knowledge situations of the use of Information and Communication Technology in educational environments face-to-face and distance learning in Higher Education Institutions.}

\author{
M.I.S.T. Miguel Ángel Romero Ochoa \\ miguelangel.romerooc@gmail.com \\ MGTI Julian Flores Figueroa \\ julian.flores.figueroa@ hotmail.com
}

\author{
M.G.T.I. Francisco Alan Espinoza Zallas \\ alanez@outlook.com
}

\begin{abstract}
Resumen
¿Cuáles son las limitantes para ampliar la cobertura de la educación?, esta es una interrogante que nos planteamos los académicos en distintos subsistemas de educación con un cierto grado de preocupación debido a las problemáticas inherentes a nuestro entorno. Afortunadamente, la evolución tecnológica y la integración de las Tecnologías de Información y Comunicación nos ha permitido ampliar nuestros horizontes en materia de cobertura en la educación de calidad, en los cuales, prácticamente no tenemos limitaciones, ya que estas facilidades nos permiten diseñar y utilizar entornos de educación virtuales, las cuales, son de gran apoyo para las distintas modalidades de educación tales como presenciales, semi-presenciales y totalmente a distancia. En cualquiera de estos casos, observamos una ventaja para la población estudiantil, ya que estas plataformas contribuyen a desarrollar el aprendizaje autónomo y autodidacta, además se fortalece el hábito de la lectura y se facilita la comunicación con los docentes ya que ésta se desarrolla de forma dinámica. Estos solo son algunos de los beneficios que se viven en la comunidad académica, sin embargo, estas modalidades también han permitido la inclusión de un segmento de la población antes
\end{abstract}


Año 11. Frontera

Núm. 28
Revista de Investigación Académica sin

ISSN: 2007-8870

\section{http://revistainvestigacionacademicasinfrontera.com}

excluida tales como ciudadanos que se encuentran laborando a tiempo completo en el sector productivo, siendo estas plataformas factor determinante para continuar con sus estudios.

El propósito de este trabajo, es presentar las tendencias actuales en el proceso de enseñanzaaprendizaje-conocimiento con relación a las Instituciones de Educación Superior. Se presenta una breve introducción sobre las Tecnologías de Información y Comunicación (TIC) y su integración en la educación. Posteriormente se explican los entornos de aprendizaje presenciales y a distancia para después hablar sobre las tendencias actuales en el uso de las TICS. Finalmente se presentan las conclusiones y referencias del documento.

Palabras Clave: Tecnologías de la Información y las comunicaciones, Entornos educativos virtuales, Entornos educativos presenciales, Modalidad a distancia, MOOC, Enseñanzaaprendizaje.

\section{Abstaract}

What are the limitations to expand the coverage of education?, This is a question that raised the academics in different subsystems of education with a degree of concern due to the problems inherent in our environment. Fortunately, the technological evolution and the integration of Information and Communication Technologies has allowed us to broaden our horizons in terms of coverage in quality education, in which we have practically no limitations, since these facilities allow us to design and use environments of virtual education, which are of great support for the different modalities of education such as face-to-face, semiface-to-face and totally distance learning. In any of these cases, we see an advantage for the student population, since these platforms contribute to the development of autonomous and self-taught learning, as well as strengthening the habit of reading and facilitating communication with teachers as it develops in a dynamic way. These are just some of the benefits that are experienced in the academic community, however, these modalities have also allowed the inclusion of a segment of the previously excluded population such as citizens who are working full time in the productive sector, being these platforms determining factor to continue with their studies.

The purpose of this document is to present current trends in the teaching-learning-knowledge process in relation to Higher Education Institutions. A brief introduction on Information and Communication Technologies (ICT) and its integration in education is presented. 
Año 11.

Frontera

Núm. 28
Revista de Investigación

Académica sin

ISSN: 2007-8870

\section{http://revistainvestigacionacademicasinfrontera.com}

Subsequently, the classroom and distance learning environments are explained, to then talk about current trends in the use of ICTs. Finally, the conclusions and references of the document are presented.

\section{Keywords}

Information and communication technologies, Virtual educational environments, Face-toface educational environments, Distance mode, The teaching-learning process.

\section{Tecnologías de Información y Comunicación}

Desde una orientación educativa, Baelo \& Cantón (2009), definen las Tecnologías de Información y Comunicación (TIC) como una práctica social que facilita los procesos de información y comunicación, gracias a los diversos desarrollos tecnológicos, en favor de una construcción y extensión del conocimiento que derive en la satisfacción de las necesidades de los integrantes de una determinada organización social.

Para Ramirez, Sabaté, Llinas, \& Lordan (2016), las TIC facilitan el procesamiento y distribución de información de datos vía electrónica por lo que se puede utilizar tecnología en el proceso de enseñanza-aprendizaje para contribuir en gran medida, a que las Universidades desarrollen y utilicen la tecnología como parte de sus procesos educativos.

\subsection{Integración de las TICS en la educación}

Las TIC han incidido en todos los ámbitos del quehacer humano; uno de ellos es la educación, lo que ha generado nuevos paradigmas y necesidades que llevan a las instituciones educativas a actualizarse en asuntos tecnológicos (Arras, Valencia, \& Tejedor, 2014). De acuerdo con la UNESCO ${ }^{1}$ (2009), el uso de las TIC en la Educación Superior puede ampliar el acceso a oportunidades de aprendizaje, mejorar sus logros y la

\footnotetext{
${ }^{1}$ Organización de las Naciones Unidas para la Educación, la Ciencia y la Cultura
} 
Año 11.

Frontera

Núm. 28
Revista de Investigación

Académica sin

ISSN: 2007-8870

\section{http://revistainvestigacionacademicasinfrontera.com}

calidad de la educación incorporando métodos avanzados de enseñanza, así como impulsar la reforma de los sistemas educativos. Pérez \& Saker (2013), mencionan que las TIC se están convirtiendo en herramientas cada vez más indispensables en las Instituciones de Educación Superior, porque sirven de apoyo didáctico: permiten intercambiar trabajos, ideas, información diversa, procesadores de texto, editores de imágenes, de páginas Web, presentaciones multimedia ${ }^{2}$, utilización de aplicaciones interactivas para el aprendizaje, recursos en páginas Web y visitas virtuales, sólo por mencionar algunas.

Por tanto, en este tipo de instituciones, las TIC cobran un importante protagonismo, conformándose como elementos esenciales para el desarrollo de la flexibilidad organizativa de las enseñanzas y el desarrollo de nuevas sinergias que conllevan plenamente a las Universidades hacia las sociedades del conocimiento. Las experiencias desarrolladas en este sentido pronostican un futuro prometedor para aquellas instituciones que integren, de forma efectiva, las TIC en sus procesos y estructuras de enseñanza ya sea en modalidad presencial o a distancia (Baelo \& Cantón, 2009).

\subsubsection{Uso de las TIC en la educación en modalidad presencial}

En el proceso de enseñanza a modalidad presencial, Arras, Valencia, \& Tejedor (2014), mencionan que las TIC se utilizan como un elemento de apoyo para la exposición de la clase, ya sea por parte del docente o de los estudiantes, de manera que se requiere encontrar nuevos modos de utilizarlas en beneficio de la formación académica universitaria tanto de los docentes como de los estudiantes.

En este tipo de modalidad de enseñanza, el uso de las TIC produce una ruptura de las limitaciones físicas del espacio 'aula', actuando como un espacio de comunicación e

\footnotetext{
${ }^{2}$ Sistema que realiza la Integración de audio, video, imagen, texto e interactividad con la finalidad de presentar información.
} 
Año 11.

Frontera

Núm. 28
Revista de Investigación Académica sin

ISSN: 2007-8870

\section{http://revistainvestigacionacademicasinfrontera.com}

intercambio de información entre los miembros de la comunidad educativa (padres, profesores, alumnos...). El uso pedagógico de las redes de comunicación puede propiciar que la relación entre educadores y educandos encuentre un ambiente que estimule la función del estudiante, como un agente activo de su propia instrucción, y la del maestro, como un guía más como una autoridad inapelable. Adicionalmente, Internet no solo provee herramientas, medios, recursos y contenidos sino, principalmente, entornos y ambientes que promueven interacciones y experiencias de interconexión e innovación educativa (Belloch, 2012).

\subsubsection{Uso de las TIC en la educación a modalidad a distancia}

El uso de las TIC como proceso de enseñanza en la modalidad a distancia se hace cada vez más popular en los entornos académicos universitarios nacionales e internacionales. Se estima que durante el año 2015 el $50 \%$ de los universitarios de todo el mundo estaban inscritos en algún curso de modalidad a distancia y tres de cada cuatro universidades usaban las TIC en sus actividades académicas (Ibercampus, 2015).

En estudios realizados por la Univertity of Oxford (2015), se menciona que las ventajas que brinda el uso de las TIC dentro de la educación a distancia es que cada vez más surgen plataformas educativas con mayores potencialidades, que proporcionan nuevas formas de diseñar e impartir la educación.

La generación de tecnologías orientadas a la educación a distancia es de suma importancia debido a que, para hacer efectivo el proceso enseñanza-aprendizaje, ésta requiere de apoyos para eliminar la separación física entre el estudiante y el tutor, por lo tanto, se necesita de las redes de cómputo que faciliten superar las distancias y constituyan una plataforma sólida para el soporte de las herramientas y materiales didácticos, cuyo contenido esté organizado atendiendo a teorías del aprendizaje y facilidades de interacción con el alumno a distancia. En este contexto, diversos organismos educativos a nivel superior se han dado a la tarea de 
Año 11.

Frontera

Núm. 28
Revista de Investigación

Académica sin

ISSN: 2007-8870

\section{http://revistainvestigacionacademicasinfrontera.com}

reflexionar en torno al uso de TIC en la educación a distancia y proponer alternativas para un mejor uso de las mismas (Garduño, 2008).

\section{Tendencia actual en el uso de las TIC en la educación superior}

Anteriormente se explicó sobre los entornos educativos en modalidad presencial y a distancia. Es importante mencionar que existen Instituciones de Educación Superior que combinan ambos entornos educativos en sus procesos de enseñanza y no solo uno de ellos. Por lo anterior es importante que el estudiante cuente con las herramientas tecnológicas que le permitan estar informado con lo relacionado a su proceso de aprendizaje.

Para Chiappe (2016), el uso de dispositivos tecnológicos que permitan llevar a cabo el proceso de aprendizaje entre los estudiantes es un componente fundamental en el desarrollo de la educación del siglo XXI. Uno de estos dispositivos es el uso de dispositivos móviles (smartphones ${ }^{3}$ o tablets ${ }^{4}$ ). Las TIC y los dispositivos móviles permiten a los integrantes de la comunidad educativa informarse, estudiar, trabajar, comunicarse e interaccionar en diversos espacios, reales y virtuales. Se rompen las fronteras del aula y se abre un gran abanico de opciones para alumnos y profesores.

Gracias a las TIC y el aprendizaje a distancia y móvil han surgido los entornos educativos digitales, accesibles en cualquier momento y lugar desde cualquier dispositivo con conexión a Internet, los cursos masivos en línea o MOOC por sus siglas en inglés, los espacios colaborativos en red, el almacenamiento y la documentación compartida en la nube ${ }^{5}$, así como la comunicación instantánea.

\section{Conclusiones}

En la actualidad, el uso de Tecnologías de Información y Comunicación ha requerido transformar los modelos educativos y métodos de enseñanza-aprendizaje-conocimiento con el propósito de que éstas sean utilizadas en forma adecuada. Como mencionó anteriormente y tomando como referencia

\footnotetext{
${ }^{3}$ Teléfono inteligente que funciona como una pequeña computadora.

${ }^{4}$ Computadora portátil, caracterizada por se de mayor volumen que un teléfono inteligente.

${ }^{5}$ Permite ofrecer Servicios de computación a través de una red que usualmente es internet.
} 
Año 11.

Frontera

Núm. 28
Revista de Investigación Académica sin

ISSN: 2007-8870

\section{http://revistainvestigacionacademicasinfrontera.com}

algunas de las situaciones presentadas, las condiciones de enseñanza de las Instituciones de Educación Superior han cambiado considerablemente y se espera que la introducción de las TIC como parte de los procesos de enseñanza atiendan las necesidades reales de maestros y estudiantes, sin importar si se utilizan en la modalidad de aprendizaje presencial o a distancia.

Los entornos de aprendizaje presencial o a distancia, los cuáles se apoyan de las TICS, están encaminados a que ahora el estudiante sea el responsable y encargado de adquirir los conocimientos, gracias a que con ayuda de herramientas tecnológicas el conocimiento se vuelve colectivo y el estudiante se hace cargo de su propia educación, interactuando con el entorno en que se desenvuelve.

\section{Referencias}

Arras, A., Valencia, K., \& Tejedor, F. (2014). Perspectiva de estudiantes de posgrado sobre escenarios de aprendizaje, condiciones de la docencia y competencias en TIC en las modalidades presencial y virtual. Revista de Innovación Educativa.

Baelo, R., \& Cantón, I. (2009). Las tecnologías de la información y la comunicación en la educación superior. Estudio descriptivo y de revisión. Revista Iberoamericana de Educación.

Belloch, C. (2012). Las Tecnologías de la Información y Comunicación en el aprendizaje. Obtenido de https://www.uv.es/bellochc/pedagogia/EVA1.pdf

Chiappe, A. (2016). Tendencias sobre contenidos educativos digitales en Amética latina. Cuaderno SITEAL.

Garduño, R. (2008). Las Tecnologías y la Educación Superior a Distancia en México. Revista Digital Universitaria.

Guzmán, T., Escudero, A., Ordaz, T., Chaparro, R., \& García, T. (2016). Principios y lineamientos de la educación a distancia, abierta y mixta de la Universidad Autónoma de Querétaro. Querétaro, Querétaro. 
Año 11.

Frontera

Núm. 28
Revista de Investigación Académica sin

ISSN: 2007-8870

\section{http://revistainvestigacionacademicasinfrontera.com}

Ibercampus. (2015). El 50\% de los universitarios de todo el mundo están inscritos en cursos de eLearning. Blog Ibercampus: El digital de la enseñanza superior europea e iberoamerica. Obtenido de Ibercampus: https://www.ibercampus.es/articulo.asp?idarticulo=30744

Pérez, M., \& Saker, A. (2013). Importancia del uso de las plataformas virtuales en la formación superior para favorecer el cambio de actitud hacia las TIC; Estudio de caso: Universidad del Magdalena, Colombia. Revista Iberoamericana de Evaluación Educativa, 153-166.

Ramirez, R., Sabaté, F., Llinas, J., \& Lordan, O. (2016). Aceptación y uso de los sistemas elearning por estudiantes de grado de ecuador: El caso de una Universidad Estatal. OmniaScience.

UNESCO. (2009). Medición de las Tecnologías de la Información y la Comunicación (TIC) en la educación. . Quebec, Montreal: UNESCO.

Univertity of Oxford. (2015). International Trends in Higher Education. University of Oxford. 